\title{
Unambiguous discrimination of mixed states
}

\author{
Terry Rudolph, ${ }^{1}$ Robert W. Spekkens, ${ }^{2,3}$ and Peter S. Turner ${ }^{3}$ \\ ${ }^{1}$ Bell Labs, 600 Mountain Ave., Murray Hill, NJ 07974, U.S.A. \\ ${ }^{2}$ Perimeter Institute for Theoretical Physics, 35 King St. North, Waterloo, Ontario N2J 2W9, Canada \\ ${ }^{3}$ Department of Physics, University of Toronto, 60 St.George Street, Toronto, Ontario M5S 1A7, Canada
}

(Dated: November 11, 2018)

\begin{abstract}
We present the conditions under which probabilistic error-free discrimination of mixed states is possible, and provide upper and lower bounds on the maximum probability of success for the case of two mixed states. We solve certain special cases exactly, and demonstrate how the problems of state filtering and state comparison can be recast as problems of mixed state unambiguous discrimination.
\end{abstract}

PACS numbers: 03.67-a

\section{INTRODUCTION}

A characteristic feature of quantum mechanics is that if a system is prepared in one of a set of non-orthogonal pure states, then there is no measurement that can yield an error-free determination of which state was prepared. Nonetheless, it is possible to achieve a probabilistic errorfree discrimination, that is, one which sometimes fails but when successful never gives an erroneous result. This sort of discrimination procedure is generally referred to as unambiguous discrimination (UD). The UD of two pure states prepared with equal prior probabilities was considered by Ivanovic and Dieks [1] and the optimal procedure was given by Peres [2]. This was generalized to the case of unequal prior probabilities by Jaeger and Shimony 3]. The problem of three pure states was analysed in 4], while multiple pure states were considered in [5, 6].

It is a common misconception that the unambiguous discrimination of mixed states is impossible [7, 8]. Indeed, in [7] it is explicitly stated that "one cannot unambiguously discriminate mixed states." That such a claim cannot be correct for an arbitrary mixed-state ensemble is proven, however, by the following counterexample: any set of orthogonal mixed states can always be discriminated with zero probability of error.

What is less obvious is that there exist sets of nonorthogonal mixed states for which UD is possible. The critical feature of such sets is that their elements do not have identical supports [16. In fact, all that is required for there to be a non-zero probability of error-free discrimination is that one of the density operators have a non-zero overlap with the intersection of the kernels of the others.

In this paper, we consider the problem of determining the optimal UD procedure for an arbitrary pair of mixed states. We derive strong upper and lower bounds on the probability of a conclusive result, and we provide an exact solution in the special case where both states have kernels of dimension 1.

The ability to unambiguously discriminate a pair of mixed states has many applications. Indeed, several recently studied problems can be recast as special cases of
UD of mixed states. We shall focus on two tasks of this sort: state comparison [9] (determining whether two systems are described by the same or different pure states) and state filtering [10,11] (discriminating one pure state from a set of pure states). We find that for the state comparison problem, our upper and lower bounds coincide, and thus automatically yield the optimal solution. For the state filtering problem, we find that our lower bound is equal to the optimal solution found in [10, 11]. Given that our lower bound has a simple geometric interpretation, it serves to clarify the nature of the optimal state filtering procedure. In particular, it shows that the eigenbases of the optimal POVM elements depend only on the subspace spanned by the states against which one is trying to filter, and not on the specific states themselves. In addition, our approach generalizes in a straightforward way to more complicated estimation tasks, such as state filtering and state comparison when the unknown states are themselves mixed.

It should be noted that our results apply not only to mixtures that arise from ignorance about which of several pure state descriptions applies, but also to those arising as the reduced density operator of an entangled state. As such, our results can be applied to the task of achieving an UD of two entangled states of a composite system given access to only one of the subsystems. It is also worth noting that our lower bound is obtained by making implicit use of the CS decomposition, which constitutes a powerful tool in both modern linear algebra and classical signal analysis 12. To our knowledge, this is the first application of the CS decomposition in quantum signal analysis.

\section{GENERAL FORMULATION}

We consider the task of discriminating unambiguously between two mixed states $\rho_{0}$ and $\rho_{1}$ with prior probabilities $p_{0}$ and $p_{1}$. The measurement procedure can have up to three outcomes, associated with identifying the state as $\rho_{0}$, identifying the state as $\rho_{1}$, and failing to identify the state conclusively. The most general threeoutcome measurement is represented in quantum mechanics by a three-element positive-operator valued mea- 
sure (POVM), which we denote by $\left\{E_{0}, E_{1}, E_{\text {? }}\right\}$. Because the identification must never be in error, we require that

$$
\operatorname{Tr}\left(\rho_{0} E_{1}\right)=\operatorname{Tr}\left(\rho_{1} E_{0}\right)=0 .
$$

The probability $P$ of successful UD is:

$$
P=p_{0} \operatorname{Tr}\left(\rho_{0} E_{0}\right)+p_{1} \operatorname{Tr}\left(\rho_{1} E_{1}\right) .
$$

We shall denote the support of $\rho_{b}$ by $\operatorname{supp}\left(\rho_{b}\right)$ and its kernel by $\mathcal{K}_{b}(b=0,1)$. It is clear that any intersection of $\mathcal{K}_{0}$ and $\mathcal{K}_{1}$ is not useful for the purposes of discriminating $\rho_{0}$ and $\rho_{1}$ since neither state has any overlap with this subspace. We shall therefore assume, in what follows, that the Hilbert space is equal to the span of the supports of $\rho_{0}$ and $\rho_{1}$. A necessary and sufficient condition for satisfying (11) is that the POVM element $E_{0}\left(E_{1}\right)$ have support only in the subspace $\mathcal{K}_{1}\left(\mathcal{K}_{0}\right)$. It follows that for there to be a non-zero probability of success, at least one of $\mathcal{K}_{0}$ and $\mathcal{K}_{1}$ must be non-zero. This occurs if and only if $\operatorname{supp}\left(\rho_{0}\right) \neq \operatorname{supp}\left(\rho_{1}\right)$.

We seek to maximize (2) subject to (11) and the constraint that $E_{0}, E_{1}$, and $E_{\text {? }}$ be positive and sum to the identity. It suffices to vary over positive $E_{0}$ and $E_{1}$ which satisfy

$$
I-E_{0}-E_{1} \geq 0 \text {. }
$$

This optimization problem is an instance of a semidefinite programming problem for which there exist efficient numerical algorithms. (See 6] for applications of semi-definite programming to UD of pure states, and [7, 8] for applications to the problem of optimizing the discrimination probability between mixed states for a fixed error rate, which interpolates between maximum likelihood estimation and UD).

\section{SOLUTIONS IN CERTAIN SPECIAL CASES}

\section{A. Mixed states with orthogonal kernels}

If $\mathcal{K}_{0} \perp \mathcal{K}_{1}$, the optimal POVM which satisfies (3) is clearly $E_{0}=K_{1}, E_{1}=K_{0}$, where $K_{b}$ is the projector onto $\mathcal{K}_{b}$. This yields a probability of success $P^{\max }=p_{0} \operatorname{Tr}\left(\rho_{0} K_{1}\right)+p_{1} \operatorname{Tr}\left(\rho_{1} K_{0}\right)$. Note that this solution also applies when one of the kernels is the null space. Note also that commuting mixed states necessarily have orthogonal kernels. It follows that this result specifies the maximum probability of UD for overlapping classical probability distributions.

\section{B. Mixed states with 1-dimensional kernels}

We now turn to the special case wherein the kernels are both 1-dimensional - that is, the states $\rho_{0}$ and $\rho_{1}$ have rank $n-1$, and the span of their supports is an $n$ dimensional space. Denoting by $\left|k_{b}\right\rangle\left\langle k_{b}\right|$ the projector onto $\mathcal{K}_{b}$, the POVM must be of the form $\left\{E_{0}=\alpha\left|k_{1}\right\rangle\left\langle k_{1}\right|, E_{1}=\right.$ $\left.\beta\left|k_{0}\right\rangle\left\langle k_{0}\left|, E_{\text {? }}=I-\alpha\right| k_{1}\right\rangle\left\langle k_{1}|-\beta| k_{0}\right\rangle\left\langle k_{0}\right|\right\}$. Our task therefore becomes to compute

$$
P^{\max }=\max _{\alpha, \beta}\left(\alpha p_{0}\left\langle k_{1}\left|\rho_{0}\right| k_{1}\right\rangle+\beta p_{1}\left\langle k_{0}\left|\rho_{1}\right| k_{0}\right\rangle\right),
$$

where the maximization is subject to the constraint

$$
I-\alpha\left|k_{1}\right\rangle\left\langle k_{1}|-\beta| k_{0}\right\rangle\left\langle k_{0}\right| \geq 0 .
$$

For convenience, we define $\theta$ to be the angle in Hilbert space between the 1-dimensional kernels,

$$
\cos ^{2} \theta \equiv\left|\left\langle k_{0} \mid k_{1}\right\rangle\right|^{2}
$$

Taking the minimum eigenvalue of the left hand side of (5), we can re-express this inequality as

$$
\frac{1}{2}\left(\alpha+\beta+\sqrt{(\alpha-\beta)^{2}+4 \alpha \beta \cos ^{2} \theta}\right) \leq 1 .
$$

Solving for $P^{\max }$ under this constraint we find that $\alpha^{\text {opt }}=\left(1-\sqrt{A_{1} / A_{0}} \cos \theta\right) / \sin ^{2} \theta, \quad \beta^{\text {opt }}=(1-$ $\left.\sqrt{A_{0} / A_{1}} \cos \theta\right) / \sin ^{2} \theta$, and

$$
P^{\max }= \begin{cases}\frac{A_{0}+A_{1}-2 \cos \theta \sqrt{A_{0} A_{1}}}{\sin ^{2} \theta} & \text { if } \cos \theta<\sqrt{\frac{A_{\min }}{A_{\max }}} \\ A_{\max } & \text { otherwise. }\end{cases}
$$

where $A_{0}=p_{0}\left\langle k_{1}\left|\rho_{0}\right| k_{1}\right\rangle, A_{1}=p_{1}\left\langle k_{0}\left|\rho_{1}\right| k_{0}\right\rangle$, and where $A_{\text {min }}=\min \left\{A_{0}, A_{1}\right\}, A_{\max }=\max \left\{A_{0}, A_{1}\right\}$. This solution, considered as a function of $\left|k_{0}\right\rangle$ and $\left|k_{1}\right\rangle$, will be denoted $P_{1 \mathrm{D}}^{\max }\left(\left|k_{0}\right\rangle,\left|k_{1}\right\rangle\right)$.

The problem of unambiguously discriminating two non-orthogonal pure states $\left|\psi_{0}\right\rangle,\left|\psi_{1}\right\rangle$ is a special case of the 1-dimensional kernel problem. Although $\cos \theta$ is defined to be the overlap of the 1-dimensional kernels, clearly $\left|\left\langle\psi_{0} \mid \psi_{1}\right\rangle\right|=\cos \theta$, and (8) becomes

$$
P^{\max }= \begin{cases}1-2 \sqrt{p_{0} p_{1}}\left|\left\langle\psi_{0} \mid \psi_{1}\right\rangle\right| & \text { if }\left|\left\langle\psi_{0} \mid \psi_{1}\right\rangle\right|<\sqrt{\frac{p_{\min }}{p_{\max }}} \\ p_{\max }\left(1-\left|\left\langle\psi_{0} \mid \psi_{1}\right\rangle\right|^{2}\right) & \text { otherwise }\end{cases}
$$

in agreement with [3]. In the case of equal prior probabilities, we have $P^{\max }=1-\left|\left\langle\psi_{0} \mid \psi_{1}\right\rangle\right|$, as expected from [2].

\section{LOWER BOUND}

We consider a strategy that achieves UD of an arbitrary pair of mixed states and which is strongly dependent on the geometrical relationship between the two subspaces $\mathcal{K}_{0}$ and $\mathcal{K}_{1}$ :

Theorem Consider two arbitrary mixed states $\rho_{0}$ and $\rho_{1}$. Denote the dimensionality of their kernels, $\mathcal{K}_{0}$ and $\mathcal{K}_{1}$ by $r_{0}$ and $r_{1}$, and assume that $r_{0} \geq r_{1}$. There exist orthonormal bases $\left\{\left|k_{b}^{j}\right\rangle\right\}_{j=1}^{r_{b}}$ for $\mathcal{K}_{b}(b=0,1)$ such that for $1 \leq j \leq r_{0}, 1 \leq i \leq r_{1}$,

$$
\left\langle k_{0}^{j} \mid k_{1}^{i}\right\rangle=\delta_{i j} \cos \left(\theta_{j}\right)
$$


where the $\theta_{j}$ are the canonical angles between $\mathcal{K}_{0}$ and $\mathcal{K}_{1}$ [10]. In this case, the expression

$$
P_{L}=\sum_{j=1}^{r_{1}} P_{1 D}^{\max }\left(\left|k_{0}^{j}\right\rangle,\left|k_{1}^{j}\right\rangle\right)+\sum_{j=r_{1}+1}^{r_{0}}\left\langle k_{0}^{j}\left|\rho_{1}\right| k_{0}^{j}\right\rangle
$$

forms a lower bound on the maximum probability of discriminating unambiguously between $\rho_{0}$ and $\rho_{1}$.

Proof The proof is constructive. Let $X_{b}$ be any $n \times r_{b}$ dimensional matrix whose orthonormal columns $\operatorname{span} \mathcal{K}_{b}$. Define an $r_{b} \times r_{b}$ unitary matrix $U_{b}$, and an $r_{0} \times r_{1}$ matrix $S$ via a singular value decomposition [13]

$$
X_{0}^{\dagger} X_{1}=U_{0} S U_{1}^{\dagger} .
$$

The matrix $S$ is of the form $S=\left[\begin{array}{l}C \\ O\end{array}\right]$, where $C$ is a diagonal matrix of the form $C=\operatorname{diag}\left(\cos \theta_{1}, \ldots, \cos \theta_{r_{1}}\right)$, $\theta_{i} \in[0, \pi / 2]$, while $O$ is an $\left(r_{0}-r_{1}\right) \times r_{1}$ matrix of 0 's. Defining $\theta_{j}=\pi / 2$ for $j>r_{1}$, and denoting by $\left|k_{b}^{i}\right\rangle$ the $i$ 'th column of $X_{b} U_{b}$, we have constructed bases for $\mathcal{K}_{0}$, $\mathcal{K}_{1}$ which satisfy $\left\langle k_{0}^{j} \mid k_{1}^{i}\right\rangle=\delta_{i j} \cos \theta_{i}$, as required in the theorem.

The measurement achieving the lower bound is associated with a POVM wherein $E_{0}=\sum_{i=1}^{r_{1}} \alpha_{i}\left|k_{1}^{i}\right\rangle\left\langle k_{1}^{i}\right|, \quad E_{1}=$ $\sum_{i=1}^{r_{0}} \beta_{i}\left|k_{0}^{i}\right\rangle\left\langle k_{0}^{i}\right|$. The constraint (3) takes the form

$$
I-\sum \alpha_{i}\left|k_{1}^{i}\right\rangle\left\langle k_{1}^{i}\left|-\sum \beta_{i}\right| k_{0}^{i}\right\rangle\left\langle k_{0}^{i}\right| \geq 0 .
$$

Since the 2 dimensional subspace spanned by $\left|k_{0}^{j}\right\rangle$ and $\left|k_{1}^{j}\right\rangle$ for $1 \leq j \leq r_{1}$ is orthogonal to all other such subspaces and is orthogonal to $\left|k_{0}^{j}\right\rangle$ for $r_{1}<j \leq r_{0}$, the constraint (13) reduces to constraints of the form (5) for $1 \leq j \leq r_{1}$, and constraints of the form $I-\beta_{j}\left|k_{0}^{j}\right\rangle\left\langle k_{0}^{j}\right| \geq 0$, for $r_{1}<j \leq r_{0}$. In this manner, we have reduced the problem to $r_{1}$ separate optimizations of the form already considered in section $\amalg$ IIB Solving each of these yields the first term on the right hand side of (111) in the theorem. The remaining $r_{0}-r_{1}$ optimizations are achieved by taking $\beta_{j}=1$, which yields the second term on the right hand side of (11).

In order to understand the geometry of the eigenbases for the POVM elements in this lower bound, it is helpful to realize that the canonical angles $\theta_{i}$ form the unique geometrical invariants describing the relationship between two subspaces. They can be defined iteratively: $\theta_{1}$ is the smallest angle between any pair of vectors drawn from $\mathcal{K}_{0}$ and $\mathcal{K}_{1}$, and $\left|k_{0}^{1}\right\rangle,\left|k_{1}^{1}\right\rangle$ are the corresponding pair of vectors. $\theta_{2}$ is the smallest such angle after these two vectors are removed, and so on. In this way, one obtains a simple geometrical picture of the measurement achieving the lower bound. We note that we have not found any example of UD wherein this lower bound is not optimal. Nonetheless, given that the eigenbases for $E_{0}, E_{1}$ depend only on the subspaces spanned by $\rho_{0}, \rho_{1}$ and not on the states themselves, there is no reason to expect it to be optimal in the general case.

\section{UPPER BOUND}

Theorem An upper bound on the maximum probability of unambiguously discriminating two mixed states, $\rho_{0}$ and $\rho_{1}$, is

$$
P^{\max } \leq \begin{cases}1-2 \sqrt{p_{0} p_{1}} F\left(\rho_{0}, \rho_{1}\right) & \text { if } F\left(\rho_{0}, \rho_{1}\right)<\sqrt{\frac{p_{\min }}{p_{\max }}} \\ p_{\max }\left(1-F\left(\rho_{0}, \rho_{1}\right)^{2}\right) & \text { otherwise, }\end{cases}
$$

where $F\left(\rho_{0}, \rho_{1}\right)=\operatorname{Tr}\left|\sqrt{\rho_{0}} \sqrt{\rho_{1}}\right|$ is the fidelity.

Proof Let $\left|\psi_{b}\right\rangle$ be a purification of $\rho_{b}$ [14]. Clearly, UD of $\left|\psi_{0}\right\rangle,\left|\psi_{1}\right\rangle$ with priors $p_{0}, p_{1}$ can be achieved with a maximal probability of success that is greater than or equal to the maximum probability of success in the UD of $\rho_{0}, \rho_{1}$ with the same priors. This follows from the fact that any discrimination procedure for the latter task serves also as a discrimination procedure for the former task. Thus,

$P^{\max }\left(p_{0} \rho_{0}, p_{1} \rho_{1}\right) \leq \min _{\left|\psi_{0}\right\rangle,\left|\psi_{1}\right\rangle} P^{\max }\left(p_{0}\left|\psi_{0}\right\rangle\left\langle\psi_{0}\left|, p_{1}\right| \psi_{1}\right\rangle\left\langle\psi_{1}\right|\right)$.

where the minimization is over all purifications of $\rho_{b}$. We know, however, that $P^{\max }\left(p_{0}\left|\psi_{0}\right\rangle\left\langle\psi_{0}\left|, p_{1}\right| \psi_{1}\right\rangle\left\langle\psi_{1}\right|\right)$ is given by Eq. (9). Minimizing either of the expressions on the right hand side of (9) requires maximizing $\left|\left\langle\psi_{0} \mid \psi_{1}\right\rangle\right|$. Uhlmann's theorem [14] states that the maximum overlap between purifications of two density operators is equal to the fidelity between the density operators. Applying this observation yields the desired upper bound.

In the case of equal prior probabilities, the upper bound has the simple form $P^{\max } \leq 1-F\left(\rho_{0}, \rho_{1}\right)$. Numerical studies of rank 2 mixed states in a 4 dimensional Hilbert space indicate that even for randomly chosen $\rho_{0}$ and $\rho_{1}$, our upper and lower bounds are generally very close.

\section{SOME APPLICATIONS}

\section{A. State comparison}

In [9], Barnett, Chefles and Jex introduced the following problem: given two systems, each of which is in one of two (generally non-orthogonal) states $\left\{\left|\psi_{1}\right\rangle,\left|\psi_{2}\right\rangle\right\}$, with what probability can one determine whether the two systems are in the same state or in different states? Here, we consider the case when this determination must be made without error if at all. Assuming equal likelihood for either possibility, we can clearly interpret the problem as one of achieving UD of the two mixed states

$$
\begin{aligned}
& \rho_{0}=\frac{1}{2}\left|\psi_{1} \psi_{1}\right\rangle\left\langle\psi_{1} \psi_{1}\left|+\frac{1}{2}\right| \psi_{2} \psi_{2}\right\rangle\left\langle\psi_{2} \psi_{2}\right| \\
& \rho_{1}=\frac{1}{2}\left|\psi_{1} \psi_{2}\right\rangle\left\langle\psi_{1} \psi_{2}\left|+\frac{1}{2}\right| \psi_{2} \psi_{1}\right\rangle\left\langle\psi_{2} \psi_{1}\right| .
\end{aligned}
$$

as was recognized by the authors of 9 .

These are rank two mixed states in a 4-dimensional space; as such their kernels are also two dimensional. We let $\left|\bar{\psi}_{i}\right\rangle$ be the state orthogonal to $\left|\psi_{i}\right\rangle$, and choose 
phases such that $\left\langle\bar{\psi}_{1} \mid \bar{\psi}_{2}\right\rangle$ is real. It is clear that $\mathcal{K}_{0}$ is spanned by $\left\{\left|\bar{\psi}_{1} \bar{\psi}_{2}\right\rangle,\left|\bar{\psi}_{2} \bar{\psi}_{1}\right\rangle\right\}$ while $\mathcal{K}_{1}$ is spanned by $\left\{\left|\bar{\psi}_{1} \bar{\psi}_{1}\right\rangle,\left|\bar{\psi}_{2} \bar{\psi}_{2}\right\rangle\right\}$. Using the techniques of section (IV), we find that the canonical basis vectors in $\mathcal{K}_{0}$ are $\left|k_{0}^{1}\right\rangle=$ $\left|\bar{\psi}_{1} \bar{\psi}_{2}\right\rangle+\left|\bar{\psi}_{2} \bar{\psi}_{1}\right\rangle,\left|k_{0}^{2}\right\rangle=\left|\bar{\psi}_{1} \bar{\psi}_{2}\right\rangle-\left|\bar{\psi}_{2} \bar{\psi}_{1}\right\rangle$, while those of $\mathcal{K}_{1}$ are $\left|k_{1}^{1}\right\rangle=\left|\bar{\psi}_{1} \bar{\psi}_{1}\right\rangle+\left|\bar{\psi}_{2} \bar{\psi}_{2}\right\rangle,\left|k_{1}^{2}\right\rangle=\left|\bar{\psi}_{1} \bar{\psi}_{1}\right\rangle-\left|\bar{\psi}_{2} \bar{\psi}_{2}\right\rangle$ (all suitably normalized). Since $\left\langle k_{0}^{2} \mid k_{1}^{2}\right\rangle=0$, these two states can be included in their respective POVM elements with weight 1 , i.e. $\alpha_{2}=1=\beta_{2}$. The remaining pair of states have overlap $\cos \theta_{1} \equiv\left\langle k_{0}^{1} \mid k_{1}^{1}\right\rangle$, and we can use the solution for 1-dimensional kernels. Performing this calculation, we find that our lower bound is $1-F\left(\rho_{0}, \rho_{1}\right)$. Since this is equal to our upper bound, we automatically know that this is optimal, and we have the optimal POVM by construction.

\section{B. State filtering}

A problem that has been considered recently by Sun, Bergou and Hillery [10] is that of unambiguously discriminating whether a state is $\left|\psi_{1}\right\rangle$ or whether it is in the set $\left\{\left|\psi_{2}\right\rangle,\left|\psi_{3}\right\rangle\right\}$ when the prior probabilities of the three states are $\eta_{1}, \eta_{2}$, and $\eta_{3}$ respectively. This task has been called state filtering. It is straightforward to see that it is an instance of UD of mixed states. Specifically, it is the problem of unambiguously discriminating

$$
\rho_{0}=\left|\psi_{1}\right\rangle\left\langle\psi_{1}\left|, \rho_{1}=\frac{\eta_{2}}{\eta_{2}+\eta_{3}}\right| \psi_{2}\right\rangle\left\langle\psi_{2}\left|+\frac{\eta_{3}}{\eta_{2}+\eta_{3}}\right| \psi_{3}\right\rangle\left\langle\psi_{3}\right|,
$$

with prior probabilities $p_{0}=\eta_{1}, p_{1}=\eta_{2}+\eta_{3}$. It is easy to find our lower bound for this problem. Since $\mathcal{K}_{1}$ is 1-dimensional, we associate it with a unique vector $\left|k_{1}\right\rangle$. The kernel $\mathcal{K}_{0}$ is 2-dimensional and so there is flexibility in the basis that diagonalizes $E_{1}$. Our lower bound dictates that we use the basis $\left|k_{0}^{1}\right\rangle,\left|k_{0}^{2}\right\rangle$, where $\left|k_{0}^{1}\right\rangle$ is the vector in $\mathcal{K}_{0}$ that is maximally parallel to $\left|k_{1}\right\rangle$, and $\left|k_{0}^{2}\right\rangle$ is the vector in $\mathcal{K}_{0}$ that is orthogonal to both $\left|k_{1}\right\rangle$ and $\left|k_{0}^{1}\right\rangle$. Our lower bound is $P_{L}=P_{1 \mathrm{D}}^{\max }\left(\left|k_{0}^{1}\right\rangle,\left|k_{1}\right\rangle\right)+\left\langle k_{0}^{2}\left|\rho_{1}\right| k_{0}^{2}\right\rangle$ Defining the canonical angle $\theta$ by $\cos \theta=\left\langle k_{1} \mid k_{0}^{1}\right\rangle$ and appealing to the geometry of the problem, we find $A_{0}=$ $p_{0}\left(1-\cos ^{2} \theta\right)$ and $A_{1}=p_{1} F\left(\rho_{0}, \rho_{1}\right)^{2} \tan ^{2} \theta$. Finally, defining $\tilde{F} \equiv F\left(\rho_{0}, \rho_{1}\right) / \sqrt{p_{0} / p_{1}}$, the lower bound takes the following form:

$$
P^{\max } \geq\left\{\begin{array}{cr}
1-p_{0} \cos ^{2} \theta-\frac{p_{1}}{\cos ^{2} \theta} F\left(\rho_{0}, \rho_{1}\right)^{2} & \tilde{F} \leq \cos ^{2} \theta \\
\left.1-2 \sqrt{p_{0} p_{1}} F\left(\rho_{0}, \rho_{1}\right)\right) & \cos ^{2} \theta \leq \tilde{F} \leq 1 \\
p_{1}\left(1-F\left(\rho_{0}, \rho_{1}\right)^{2}\right) & \tilde{F} \geq 1
\end{array}\right.
$$

This coincides precisely with the optimal solution derived in [10]. Thus, our lower bound is found to be optimal in this case.

By recasting state filtering as an instance of UD of a pure state and a mixed state, it has become apparent that the particular ensemble of states from which the mixed state is formed (in this case, $\left|\psi_{2}\right\rangle$ and $\left|\psi_{3}\right\rangle$ with priors $\eta_{2}$ and $\eta_{3}$ ) is not significant. As such our analysis applies also to the case wherein the second set of states contains an arbitrary number of elements, in agreement with the solution found in 11]. Moreover, the mixed state need not even arise as a result of ignorance of the pure state, but rather may arise as a result of the system being entangled with another degree of freedom. This is confirmed by the fact that the probability of success depends only on $\rho_{1}$. The problem of UD between multiple sets of pure states [15] is a straightforward generalization of state filtering that can be recast as a problem of UD of mixed states in a similar way.

\section{CONCLUSION}

We have shown that UD of a pair of mixed states is possible when these have distinct supports. We have provided the maximum probability of success for the case of mixed states with orthogonal or 1-dimensional kernels. In the general case, we have determined an upper bound based on the probability of UD for purifications of the states, and a lower bound based on the geometrical invariants between their kernels. In addition to the tasks discussed here, there are likely to be many others for which UD of mixed states is useful, such as quantum random access codes, quantum oblivious transfer and entanglement distillation, to name a few.

\section{Acknowledgments}

We thank F. Verstraete for useful discussions, and S. Salmi for logistical support. TR is supported by the NSA \& ARO under contract No. DAAG55-98-C-0040. RWS and PST are supported in part by NSERC and the Sumner Foundation. RWS and PST wish to unambiguously distinguish themselves from TR, whose support is orthogonal to their own.
[1] I. D. Ivanovic, Phys. Lett. A 123, 257 (1987); D. Dieks, Phys. Lett. A 126, 303 (1988).

[2] A. Peres, Phys. Lett. A 128, 19 (1988).

[3] G. Jaeger and A. Shimony, Phys. Lett. A 197, 83 (1995)

[4] A. Peres and D. Terno, J. Phys. A 31, 7105 (1998); Y. Sun, M. Hillery, and J. Bergou, Phys. Rev. A 64, 022311 (2001)

[5] A. Chefles, Phys.Lett. A 250, 223 (1998).
[6] Y.C. Eldar, IEEE Trans. Inform. Theory 49, 446 (2003).

[7] J. Fiurášek and M. Ježek, Phys. Rev. A 67, 012321 (2003).

[8] Such a claim was made in Y. C. Eldar, quant-ph/0211121 Based on a preprint version of the present paper, the error was recognized and corrected in the published version: Y.C. Eldar, Phys. Rev. A 67, 042309 (2003). 
[9] S. M. Barnett, A. Chefles, and I. Jex, Phys. Lett. A 307, 189 (2003).

[10] Y. Sun, J. Bergou, and M. Hillery, Phys. Rev. A 66, 032315 (2002).

[11] J. Bergou, U. Herzog, and M. Hillery, eprint quant-ph 0209007

[12] J.M. Speiser and C.F. van Loan, Proc. SPIE Vol. 495, Real Time Signal Processing 4755 (1984).
[13] R. Bhatia, Matrix Analysis (Springer-Verlag, New York, 1997).

[14] R. Josza, J. Mod. Opt. 41, 2315 (1994).

[15] S. Zhang and M. Ying, Phys. Rev. A 65, 062322 (2002).

[16] The support of a mixed state is the space spanned by its eigenvectors; the kernel is the space orthogonal to its support. 